\title{
NOS1 expression promotes proliferation and invasion and enhances chemoresistance in ovarian cancer
}

\author{
ZHIWEI ZOU ${ }^{1 *}$, XIAOXUAN LI ${ }^{2 *}$, YONGLIANG SUN ${ }^{1}$, LINLIN LI $^{1}$, QIANBING ZHANG $^{1}$, \\ LINGQUN ZHU ${ }^{1}$, ZHUO ZHONG ${ }^{1}$, MENG WANG ${ }^{1}$, QIANLI WANG ${ }^{1}$, ZHENJUN LIU $^{3}$, YIFENG WANG ${ }^{2}$, \\ YIFANG PING $^{4}$, KAITAI YAO ${ }^{1}$, BINGTAO HAO ${ }^{1}$ and QIUZHEN LIU $^{1}$
}

${ }^{1}$ Cancer Research Institute, Southern Medical University, Guangzhou, Guangdong 510515; ${ }^{2}$ Department of Obstetrics and Gynecology, Zhujiang Hospital, Southern Medical University, Guangzhou 510280; ${ }^{3}$ Department of Vascular Surgery, Nanfang Hospital, Southern Medical University, Guangzhou, Guangdong 510515; ${ }^{4}$ Institute of Pathology and Southwest Cancer Center, Southwest Hospital, Third Military Medical University, Chongqing, Sichuan 400038, P.R. China

Received January 14, 2016; Accepted July 3, 2018

DOI: $10.3892 / \mathrm{ol} .2020 .11355$

\begin{abstract}
Nitric oxide (NO), an important chemical messenger, serves a dual role in tumor progression. Nitric oxide synthase isoform 1 (NOS1) was observed to be increasingly expressed in various types of cancer, and its expression has been associated with tumor progression. However, the level of NOS1 expression and the associated functions of NOS1 in human ovarian cancer remain undefined. Using gene expression profiles of ovarian cancer from the Gene Expression Omnibus (GEO) database, the present study revealed that NOS1 was increasingly expressed in ovarian cancer tissues. The present study investigated the level of NOS1 expression and its effects on in vitro cell function, including proliferation, migration and invasion as well as chemoresistance to cispatin (DDP) treatment in OVCAR3 cells. Reverse transcription-quantitative polymerase chain reaction demonstrated that the level of NOS1 mRNA expression varied in different ovarian cancer lines. However, immunoblotting indicated that the level of NOS1 protein expression was constitutively high in ovarian cancer cell lines. Treatment with NOS inhibitor NG-nitro-L-arginine methyl ester or transfection with NOS1 short hairpin RNA significantly inhibited cell proliferation, migration and invasion compared with the control, whereas the sensitivity of OVCAR3 cells to DDP treatment was increased. The results of the present study indicated that NOS1 promoted the function of ovarian cancer cells, including proliferation, invasion and chemoresistance, providing a potential target for ovarian cancer therapeutic.
\end{abstract}

Correspondence to: Professor Qiuzhen Liu, Cancer Research Institute, Southern Medical University, 1838 North Guangzhou Road, Guangzhou, Guangdong 510515, P.R. China

E-mail: liuqiuzhen@126.com

*Contributed equally

Keywords: ovarian cancer, nitric oxide synthase isoform 1, proliferation, migration, invasion, chemoresistance

\section{Introduction}

Nitric oxide (NO) is one of the most important messengers for a number of physiological processes, including vasodilation, smooth muscle relaxation, inhibition of platelet aggregation and regulation of neurotransmission (1). Endogenous NO, synthesized from L-arginine via a family of nitric oxide synthases (NOSs), which comprises neuronal NOS (nNOS; NOS1), inducible NOS (iNOS; NOS2) and endothelial NOS (eNOS; NOS3). NOS1 and NOS3 are constitutive and produce short bursts of NO for signaling or messenger functions in a calcium-dependent manner, whereas NOS2 is expressed in response to immunological stimuli and is capable of sustained release of NO in a calcium-independent manner (2).

The production of NO and expression of NOS are ubiquitous in malignant tumors and possess both pro-tumor and antitumor effects (3). One of the primary determinants that account for the paradoxical behavior of NO in tumor biology are its concentration in tumor milieu, exposure time and cellular adaptation to NO. Generally, NO favors cell survival and proliferation at lower NO concentrations $(<100 \mathrm{nM})$. By contrast at a higher level of $\mathrm{NO}(>300 \mathrm{nM})$, NO promotes cell cycle arrest, apoptosis and senescence $(4,5)$. Therefore, higher concentrations produced by NOS2, a potential cytostatic/cytotoxic factor in immune functions, mediate antitumor activity, whereas chronic induction of NOS2 may contribute to the initiation of cancer (6). Furthermore, low concentrations of NO produced by NOS1 and NOS3 facilitated tumor progression by modulating cancer-associated events, including angiogenesis, apoptosis, cell cycle, invasion and metastasis (7). Among the three NOSs, NOS1 was also observed to be aberrantly expressed in human tumors, including the brain (8), lung (9) and glioma (10). Despite the discrepancies regarding the concentration of NO and its cellular effects, a study by Kotake et al (11) has reported that low levels of NO formed by NOS1, triggers cell proliferation primarily via the soluble guanylate cyclase-cyclic guanosine monophosphate (sGC-cGMP) dependent mechanism. Furthermore, NOS1 expression in melanoma mediated the dysfunction of response to adoptive $\mathrm{T}$ cell therapy (12). 
Previous studies suggested that NOS isoforms were highly expressed in ovarian cancer $(13,14)$. The function of NOS isoforms on ovarian tumor development is highly complex, with both tumor-promoting and inhibiting actions having been described (15). It has also been demonstrated that the level of NOS2 expression was associated with differential status, whereas NOS1 and NOS3 were mainly expressed in poorly differential samples (14). Previously, it was reported that NOS expression was associated with responsiveness of DDP treatment. The level of NOS1 expression was associated with DDP-resistance, whereas NOS2 was highly expressed in sensitive ovarian cancer cell lines (16). These results indicated that the expression of NOS isoforms serve a critical role in the progression of ovarian cancer and have an effect on the sensitivity of chemotherapy. However, the functional role of individual NOSs, particularly NOS1, on the biological behaviors of ovarian cancer remains unclear.

The present study analyzed the gene expression profiles of ovarian cancer downloaded from the Gene Expression Omnibus (GEO) database and revealed that there was a higher expression of NOS1 in ovarian cancer tissues compared with normal ovarian tissues. Using the NOS inhibitor NG-nitro-L-arginine methyl ester (L-NAME) or NOS1 knockdown by short hairpin (sh)RNA, the present study verified that NOS1 serves multiple functions in the promotion of tumor development, including proliferation, migration and invasion, as well as drug resistance in OVCAR3 cells. The results of the present study provide a suggestion for the improvement of ovarian cancer therapy.

\section{Materials and methods}

Chemicals and reagents. Unless otherwise stated, all chemicals were purchased from Sigma-Aldrich (Merck KGaA, Darmstadt, Germany).

GEO database. Analysis of gene expression profiles of NOS isoforms in ovarian cancer tissues. Expression data was downloaded from GEO (accession no. GSE14407).

Cell culture and transfection. Ovarian cancer cells lines of OVCAR3, SKOV3 and ES-2 were obtained from Southern Medical University Cancer Institute (Guangzhou, China). Cells were grown in Dulbecco's modified Eagle's medium supplemented with $10 \%$ fetal bovine serum (FBS) and $1 \%$ penicillin-streptomycin (Invitrogen; Thermo Fisher Scientific, Inc., Waltham, MA, USA). The cells were incubated at $37^{\circ} \mathrm{C}$ in a $95 \%$ air $-5 \% \mathrm{CO}_{2}$ gas mixture. The medium was replaced every 2 days. OVCAR3 Sh-NOS1 cells were transfected with NOS1 shRNA (GeneCopoeia, Guangzhou, China). A nonspecific control was used as non-targeting shRNAs. Transfections were performed using Lipofectamine 2000 reagent (Invitrogen; Thermo Fisher Scientific, Inc.) using 1-2 mg of expression vector/ml serum-free medium as described by the manufacturer. The transfected cells were incubated at $37^{\circ} \mathrm{C}$ for $24 \mathrm{~h}$ and harvested for reverse transcription-quantitative polymerase chain reaction (RT-qPCR) and western blot analysis.

$R T$-qPCR. Total cellular RNA from cells was extracted using TRIzol ${ }^{\circledR}$ reagent (Life Technologies; Thermo Fisher
Scientific, Inc.), according to the manufacturers' protocol. cDNA was synthesized from $1 \mu \mathrm{g}$ total RNA using the RNeasy mini kit (Qiagen GmbH, Hilden, Germany). cDNA was amplified using the KAPA SYBR Fast universal qPCR kit (Tiangen Biotec Co., Ltd., Beijing, China) using the following primers: Human NOS1 forward, 5'-CAGAGG ATGGCAGTCTGTTTC-3' and reverse, 5'-CTCAAGAGC ACTGGATCTCAG-3'; human GAPDH forward, 5'-TGT GGGCATCAATGGATTTGG-3' and reverse, 5'-ACACCA TGTATTCCGGGTCTTA-3'. The reaction time was as follows: $2 \mathrm{~min}$ at $95^{\circ} \mathrm{C}$ for initial denaturation, $30 \mathrm{sec}$ at $95^{\circ} \mathrm{C}$ and $32 \mathrm{sec}$ at $60^{\circ} \mathrm{C}$ for 40 cycles. Gene expression levels were normalized to those of GAPDH. RT-qPCR was performed using the Mx3005p system (Stratagene; Agilent Technologies, Inc., Santa Clara, CA, USA). Relative quantification of mRNA was determined by the $2^{-\Delta \Delta \mathrm{Cq}}$ method (17). The experiments were done at least thrice independently and all samples were in triplicate.

Western blot analysis. The harvested cells were lysed with lysis buffer containing $50 \mathrm{mM}$ Tris/ $\mathrm{HCl}(\mathrm{pH} 8.0), 150 \mathrm{mM} \mathrm{NaCl}$, $1 \%$ NP-40, $0.5 \%$ sodium deoxycholate, $0.1 \%$ SDS, $50 \mathrm{mM}$ $\mathrm{NaF}, 1 \mathrm{mM} \mathrm{Na} \mathrm{VO}_{4}$ and protease inhibitor (Hangzhou Fude Biological Technology Co., Ltd., Hangzhou, China). Protein concentrations in the cell lysates were quantified using the BCA Protein Assay kit (Beyotime Institute of Biotechnology, Haimen, China). Proteins $(50 \mu \mathrm{g})$ were separated on $10 \%$ SDS-PAGE gels and transferred to a nitrocellulose membrane (EMD Millipore, Billerica, CA, USA). Following blocking with $5 \%$ bovine serum albumin in TBS supplemented with $1 \%$ Tween- 20 at $28^{\circ} \mathrm{C}$ for $1 \mathrm{~h}$, the membranes were incubated with the appropriate primary antibodies: NOS1 (cat. no. ab76067; 1:1,000; Abcam, Cambridge, UK), NOS2 (cat. no. ab15323; 1:1,000; Abcam), NOS3 (cat. no. ab76198; 1:1,000; Abcam) and GAPDH (cat. no. G9545; 1:5,000; Sigma Aldrich; Merck $\mathrm{KGaA}$ ), at $4{ }^{\circ} \mathrm{C}$ overnight, followed by $2 \mathrm{~h}$ incubations at room temperature with horseradish peroxidase-conjugated goat anti-rabbit IgG secondary antibody (cat. no. FD0128; 1:5,000; Hangzhou Fude Biological Technology Co., Ltd.) and goat anti-mouse IgG secondary antibody (cat. no. FDM007; 1:5,000; Hangzhou Fude Biological Technology Co., Ltd.). The protein bands were visualized using an Enhanced Chemiluminescence Western Blot Detection system.

Griess assay. OVCAR3 cells were treated with NOS extensive inhibitor [NG-nitro-L-arginine methyl ester (L-NAME)] $(1 \mathrm{mM})$ and NO donor [DETA-NONOate, (DETA-NO)] $(50 \mu \mathrm{M})$ for $24 \mathrm{~h}$. Accumulation of NO in cultured media samples was analyzed by Griess assay-through measuring levels of nitrite expression using a nitrate/nitrite colorimetric assay kit. Briefly, OVCA3 cells were seeded in 96-well plates at a density of 5,000 cells/well, and then supernatant of the cultured cells was collected following the indicated time. The culture supernatant $(20 \mu \mathrm{l})$ was mixed with $60 \mu \mathrm{l}$ assay buffer, $10 \mu \mathrm{l}$ enzyme cofactor and $10 \mu \mathrm{l}$ nitrate reductase. Following incubation for $10 \mathrm{~min}$ at room temperature, $10 \mu \mathrm{DAN}$ and $20 \mu \mathrm{l} \mathrm{NaOH}$ was added to each well and the fluorescence was determined at an excitation wavelength of $365 \mathrm{~nm}$ and an emission wavelength of $430 \mathrm{~nm}$. The amount of nitrite was evaluated using a $\mathrm{NaNO}_{2}$ standard curve. 

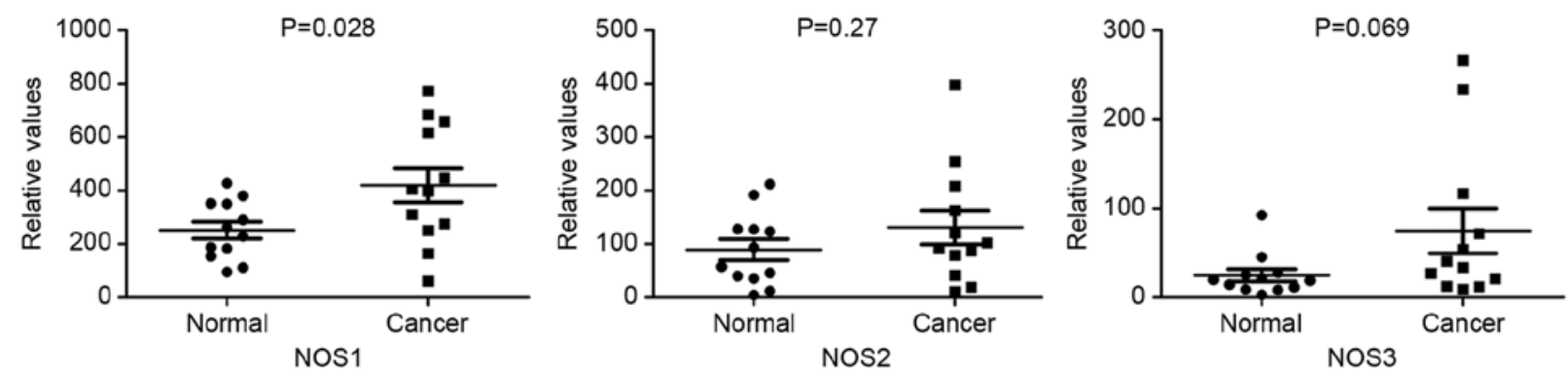

Figure 1. Analysis of gene expression profiles of NOS isoforms in ovarian cancer tissues. Expression data was downloaded from Gene Expression Omnibus (accession no. GSE14407). The relative expression level of mRNA of three NOS isoforms was higher in ovarian cancer tissues compared with normal ovarian tissues. However, only the alteration of NOS1 expression was statistically significant $(\mathrm{P}<0.05)$. NOS, nitric oxide synthase.

Cell proliferation assay. The cells were seeded into 96-well plates at a density of 5,000 cells/well and then incubated at $37^{\circ} \mathrm{C}$ with $5 \% \mathrm{CO}_{2}$. Following $24 \mathrm{~h}, 10 \mu 1 \mathrm{MTT}(5 \mathrm{mg} / \mathrm{ml})$ was added to each well and the cells were further incubated at $37^{\circ} \mathrm{C}$ for $4 \mathrm{~h}$. Subsequently, all supernatant was discarded, $100 \mu \mathrm{l}$ DMSO was added to each well and 96-well plates were agitated for $15 \mathrm{~min}$ at room temperature. The absorbance of cells was analyzed at $490 \mathrm{~nm}$ using a microplate reader.

Colony formation assay. The cells were trypsinized, counted and plated in 6-well plates at a density of 500 cells per well. Following two weeks, the cells were washed with PBS, fixed in $10 \%$ methanol for $15 \mathrm{~min}$ and stained with Giemsa for $10 \mathrm{~min}$ at room temperature. The colonies were then imaged and counted with a light microscope. All experiments were performed in triplicate.

Scratch-wound assay. The cells at a density of $5 \times 10^{5}$ cells/well were seeded into a six-well plate and cultured to $80 \%$ confluence in medium supplemented with $10 \% \mathrm{FBS}$ at $37^{\circ} \mathrm{C}$. Cell monolayers were scratched using a plastic tip $(1 \mathrm{~mm})$ and incubated in serum-containing medium (2\% serum) for $24 \mathrm{~h}$ at $37^{\circ} \mathrm{C}$. The migration distance of the cells was determined at three sites using Photoshop (Adobe Systems, Inc., San Hose, CA, USA).

Invasion assay. The invasive potential of the cancer cells was assessed in vitro using Transwell chambers (Corning Incorporated, Corning, NY, USA). The upper chambers were coated with Matrigel (BD Biosciences, Franklin Lakes, NJ, USA). Subsequently $1 \times 10^{5}$ cells in serum-free medium were added to the upper chambers and 10\% FBS was added to the bottom chambers. Cells were incubated for $24 \mathrm{~h}$ at $37^{\circ} \mathrm{C}$. The cells on the lower side of the filter were fixed with $75 \%$ methyl alcohol for $15 \mathrm{~min}$ at room temperature and where then stained with hematoxylin for $10 \mathrm{~min}$ at room temperature, and counted under a fluorescence microscope in 5 different fields (x400 magnification). Equal amounts of PBS were used as the control.

Statistical analysis. All experiments were repeated at least three times. The results are presented as the mean \pm standard deviation. The statistical significance of differences was determined by Student's t-test when comparing two groups, and one-way analysis of variance was used to compare multiple groups. $\mathrm{P}<0.05$ was considered to indicate a statistically significant difference. All data were analyzed using SPSS version 13.0 software (SPSS, Inc., Chicago, IL, USA).

\section{Results}

Expression of NOS1 in ovarian cancer. In order to evaluate the effect of NOS1 on ovarian cancer progression, the present study first analyzed the expression level of NOS isoforms, NOS1, NOS2 and NOS3, in gene expression profiles including 12 pairs of ovarian cancer tissues and normal ovarian tissues downloaded from the GEO database (http://www.ncbi.nlm. nih.gov/geo/, accession no. GSE14407). Expression levels of NOS isoforms mRNA were revealed to be increased in ovarian cancer tissues compared with normal ovarian tissues. However, only the alteration of NOS1 expression level was statistically significant (Fig. 1). The level of NOS1 expression was also increased in the DDP-resistant group compared with the DDP-sensitive group (data not shown). These results indicated that NOS1 may serve an important role in ovarian cancer progression.

Production of NO by NOS1 in ovarian cancer cells. To verify the role of NOS1 in ovarian cancer, the present study evaluated the expression levels of three NOS enzymes in a human ovarian cancer line by RT-qPCR and western blot analysis. The mRNA expression levels of the three NOSs were detectable in all analyzed ovarian cancer cell lines: SKOV3, OVCAR3 and ES-2 (Fig. 2A). Protein expression of three NOSs was also detected in SKOV3 and OVCAR3 (Fig. 2B). Subsequently, the present study treated OVCAR3 cells with NOS extensive inhibitor [NG-nitro-L-arginine methyl ester (L-NAME)] and NO donor [DETA-NONOate, (DETA-NO)] for $24 \mathrm{~h}$, and examined nitrates (NOx) in the culture medium by Griess assay. The concentration of NO released by OVCAR3 was relatively low and the value of was $\sim 24 \mathrm{nM}$ compared with the concentration released by NO donor DETA-NO $50 \mu \mathrm{M}$. The cells treated with $50 \mu \mathrm{M}$ DETA-NO released $50 \mathrm{nM}$ NO (Fig. 2C).

NOS1 promotes proliferation of OVCAR3 cells. In order to investigate the role of NOS1 in cell proliferation, the present study first investigated the effect of NOSs inhibitor L-NAME on cell proliferation using MTT and colony formation assays. The results revealed that cells treated with L-NAME demonstrated a 
A

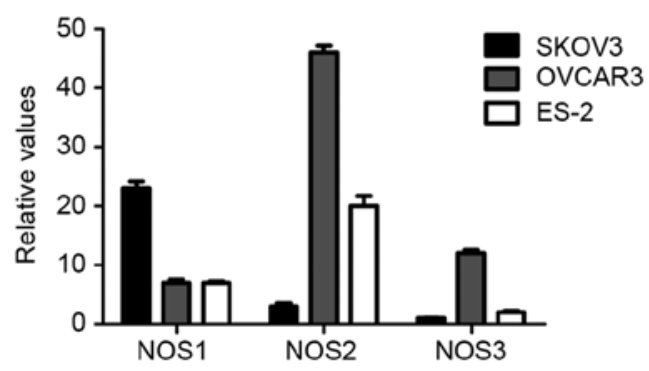

B

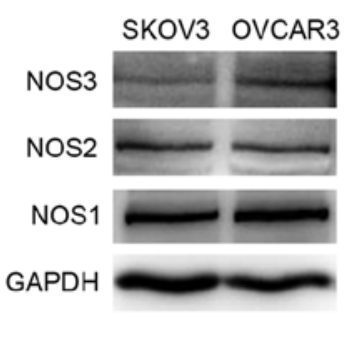

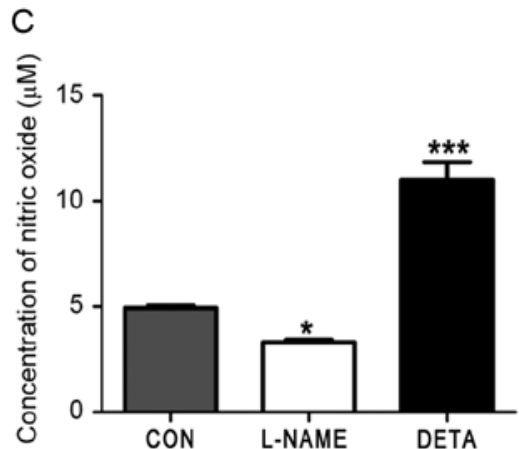

Figure 2. Analysis of mRNA and protein expression of NOS isoforms, and NO production in ovarian cancer cell lines. (A) mRNA expression of NOS isoforms were analyzed by reverse transcription-quantitate polymerase chain reaction, and expression varied in OVCAR3, SKOV3 and ES-2 cells. The levels of NOS mRNA expression were normalized to GAPDH expression. (B) The proteins of the three NOS isoforms were all detected by western blotting in SKOV3 and OVCAR3 cells. (C) The levels of NO expression in the culture medium of OVCAR3 cells was determined by Griess assay and was less than half of the concentration released by cells treated with $50 \mu \mathrm{M}$ DETA-NONOate $(50 \mathrm{nM} \mathrm{NO})$. Error bars represent the mean \pm standard deviation of triplicate experiments. ${ }^{*} \mathrm{P}<0.05$ and ${ }^{* * * *} \mathrm{P}<0.001$. All compared with control. CON, control; DETA, DETA-NONOate; L-NAME, NG-nitro-L-arginine methyl ester; NOS, nitric oxide synthase; NO, nitric oxide.

A
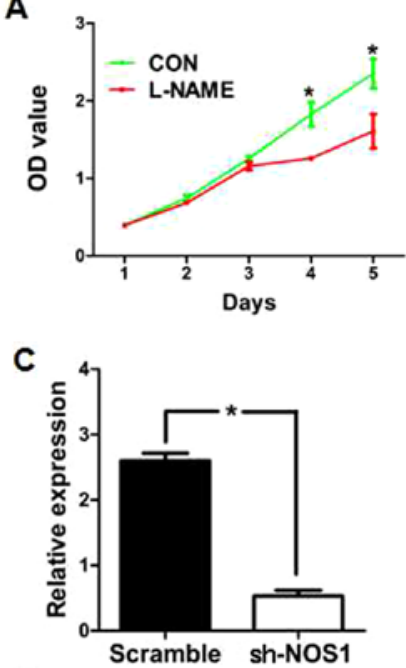

E

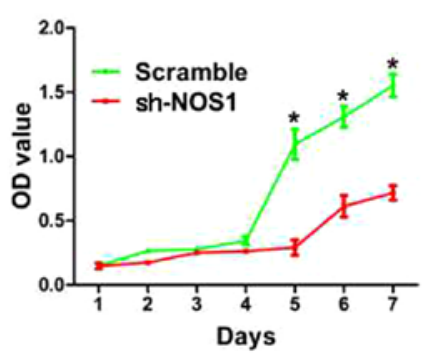

B
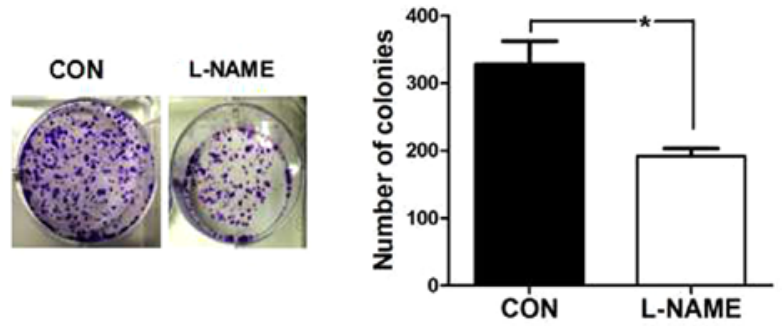

D

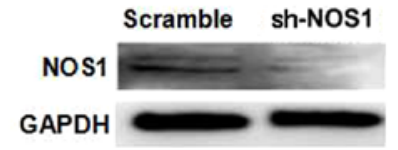

$\mathbf{F}$

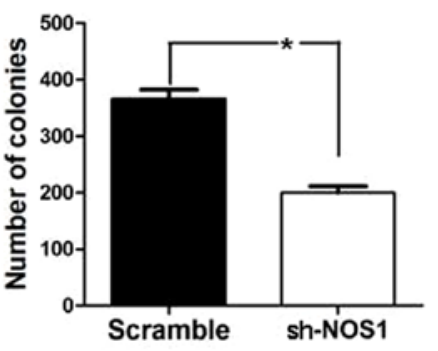

Figure 3. NOS1 expression promotes proliferation in OVCAR3 cells. L-NAME inhibited proliferation of OVCAR3 cells as determined by (A) MTT and (B) colony formation assays. " $\mathrm{P}<0.05$ vs. control. The shRNA assay revealed that the levels of mRNA (C and D) protein expression of NOS1 were markedly decreased compared with the corresponding control in OVCAR3 cells. " $\mathrm{P}<0.05$. NOS1 knockdown inhibited proliferation of OVCAR3 cells as determined by (E) MTT and (F) colony formation assays. Bars represent the mean \pm standard deviation of three independent tests. ${ }^{*} \mathrm{P}<0.05$. sh, short hairpin; CON, control; NOS, nitric oxide synthase; OD, optical density; L-NAME, NG-nitro-L-arginine methyl ester.

significantly lower proliferation rate compared with the control cells (Fig. 3A and B). Subsequently, the present study examined the effect of shRNA-mediated NOS1 knockdown on cell proliferation. OVCAR3 cells were either transfected with nonspecific shRNA (scramble) or NOS1 shRNA (Sh-NOS1). Following transfection for $24 \mathrm{~h}$, RT-qPCR and western blotting were performed to analyze the levels of NOS1 mRNA and protein expression. As presented in Fig. 3C and D, the expression of NOS1 mRNA and protein was markedly decreased in NOS1 shRNA-transfected cells compared with the control shRNA-transfected cells $(\mathrm{P}<0.01)$. The results of the MTT and colony formation assays demonstrated that NOS1 shRNA-transfected cells revealed a significantly lower proliferation rate compared with the control shRNA-transfected cells (Fig. 3E and F). 

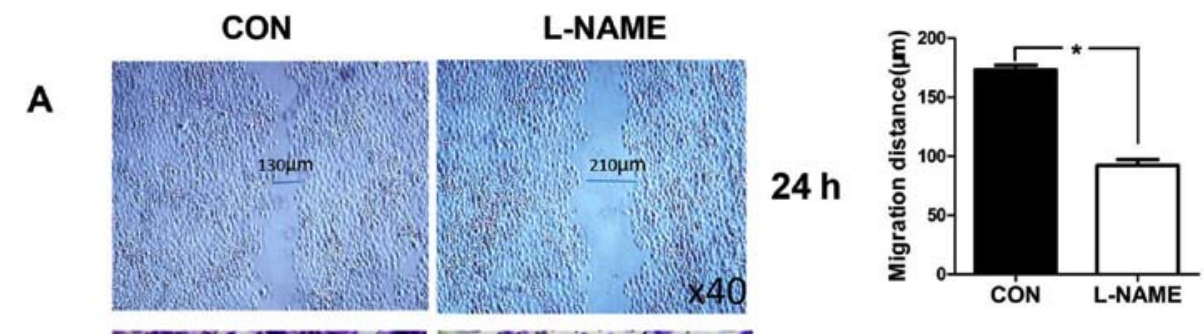

B
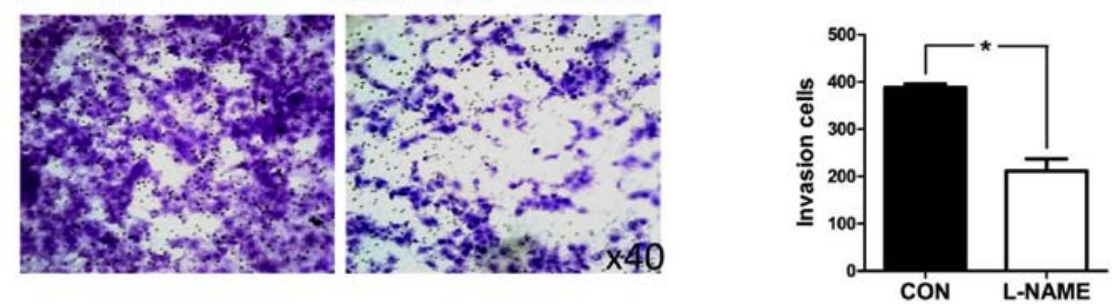

Scramble

Sh-NOS1
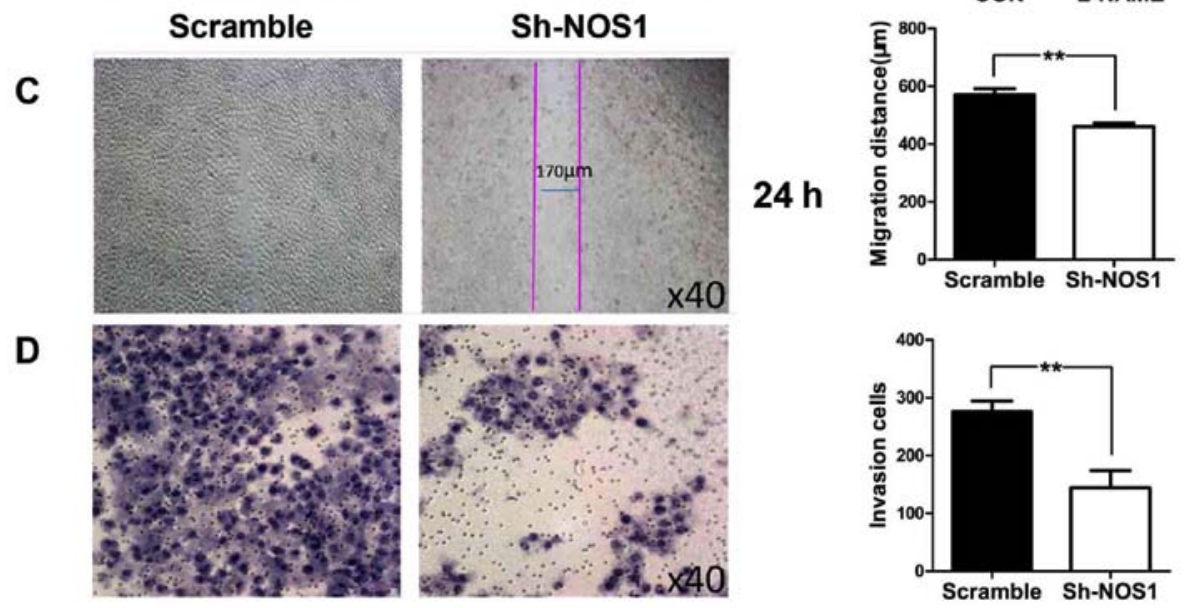

Figure 4. NOS1 promotes invasion and migration of OVCAR3 cells. (A) The migratory ability of OVCAR3 cells was inhibited by L-NAME (1 mM; 24 h) as determined by scratch wound assay. (B) L-NAME (1 mM) inhibited invasive ability of OVCAR3 cells as determined by invasion assays. NOS1 knockdown inhibited (C) migratory and (D) invasive abilities of OVCAR3 cells as determined by scratch-wound assay and invasion assays, respectively. Data is presented as the mean \pm standard deviation of three independent tests ( 40 magnification). ${ }^{*} \mathrm{P}<0.05$ and ${ }^{* *} \mathrm{P}<0.01$. CON, control; L-NAME, NG-nitro-L-arginine methyl ester; NOS, nitric oxide synthase.
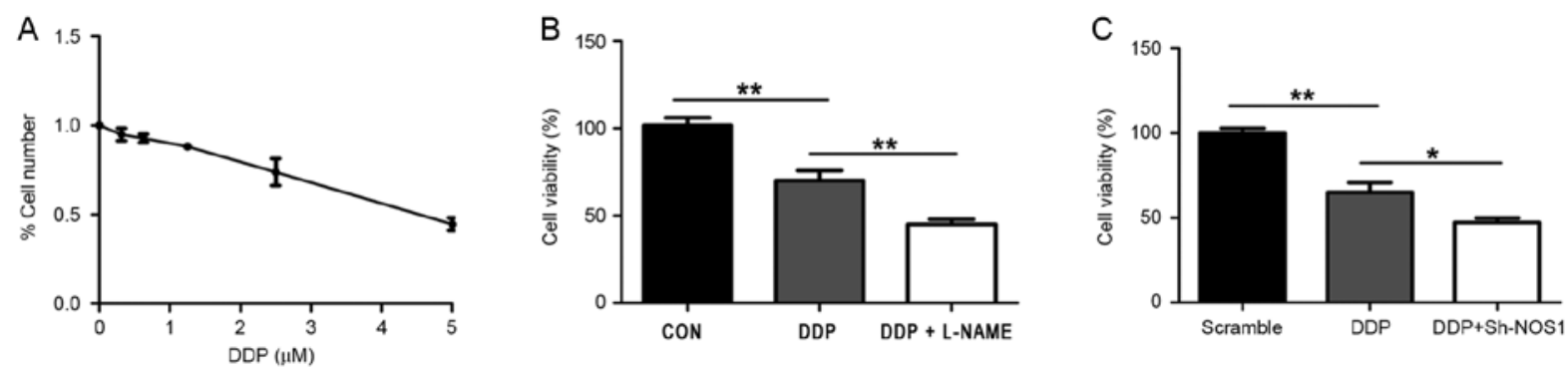

Figure 5. NOS1 activity inhibition or shRNA-mediated NOS1 knockdown increases the sensitivity of OVCAR3 cells to DDP treatment. (A) Cytotoxic curve, as determined by MTT assay, indicated the $\mathrm{IC}_{50}$ of cisplatin for OVCAR3 cells was $5 \mu \mathrm{M}$. (B) MTT assay indicated that the cytotoxicity of OVCAR3 cells to DDP treatment was increased by treatment with NOS inhibitors. (C) MTT assay demonstrated that NOS1 knockdown also decreased the viability of OVCAR3 cells in response to DDP treatment. Data is presented as the mean \pm standard deviation of three independent tests. ${ }^{*} \mathrm{P}<0.05$ and ${ }^{* *} \mathrm{P}<0.01$. DDP, cisplatin; shRNA, short hairpin RNA; L-NAME, NG-nitro-L-arginine methyl ester; NOS, nitric oxide synthase.

NOS1 promotes invasion and migration of OVCAR3 cells. The present study assessed the effect of NOS1 on tumor cell metastasis. Cellular migration was analyzed by scratch-wound assay and invasion was analyzed by Transwell assay. The results of scratch-wound assay demonstrated that NOSs inhibitor exhibited a significant decrease in cellular migration compared with the control $(\mathrm{P}<0.05$; Fig. $4 \mathrm{~A})$. In the in vitro invasion assays, the number of cells invaded through the Transwell membrane in the L-NAME-treated group was significantly lower compared with the control ( $\mathrm{P}<0.05$; Fig. 4B). Subsequently, the present study determined the effect of shRNA-mediated NOS1 knockdown on cell invasion ability in OVCAR3, respectively. The results of the scratch-wound assay demonstrated a significant reduction in motility of NOS1 shRNA-transfected cells compared with the control $(\mathrm{P}<0.01 ;$ Fig. $4 \mathrm{C})$. In the in vitro invasion assays, the number of cells invaded through the 
Transwell membrane in NOS1 shRNA-transfected group was significantly lower compared with the control $(\mathrm{P}<0.01$; Fig. 4D).

NOS1 inhibition increases sensitivity of OVCAR3 to $D D P$-induced cell death. The present study investigated the role of NOS1 in chemoresistance in ovarian cancer. Primarily, the cytotoxic effect of DDP on ovarian cancer cells (OVCAR3) analyzed by MTT assay demonstrated cytotoxic activity with an $\mathrm{IC}_{50}$ value of $5 \mu \mathrm{mol} / 1$ (Fig. 5A). Next, the effect of NOSs inhibitor combined with DDP $(2 \mu \mathrm{mol} / \mathrm{l})$ on cell proliferation was evaluated by analyzing cell viability. The results revealed that the combination of L-NAME treatment and DDP-mediated knockdown effectively promoted cell death of OVCAR3 cells compared with treatment with DDP alone (Fig. 5B). Similarly, NOS1 knockdown significantly reduced cell viability compared with treatment with DDP alone (Fig. 5C).

\section{Discussion}

Epithelial ovarian cancer has the highest mortality rate of all gynecological malignancies and is the fifth leading cause of cancer mortality in females $(18,19)$. Current therapeutic approaches for ovarian cancer are relatively effective for early-stage disease with 5-year survival rates of $84 \%$ for stage I and $66 \%$ for stage II disease, whereas the 5-year survival rates for stage III or IV disease are $<30 \%(20,21)$. More than two-thirds of females with advanced-stage epithelial ovarian cancer experience recurrence despite achieving clinical remission following completion of initial treatment (22). Chemoresistance to standard treatment is crucial for recurrence of ovarian cancer. Therefore, understanding the molecular mechanism underlying ovarian cancer development and providing effective predictive markers for recurrence or chemoresistance are urgently required for more effective management and for developing ovarian cancer therapies. The present study analyzed the gene expression profiles of ovarian cancer downloaded from the GEO database and revealed that NOS1 expression level was higher in ovarian cancer compared with paired normal ovarian tissues. The in vitro experiment verified that NOS1 promoted proliferation, invasion and migration, as well as chemoresistance in ovarian cancer cells. The present study provides a candidate marker for prognosis of ovarian cancer and an implication for improvements in ovarian cancer therapy.

Endogenous NO is produced in mammalian cells by the three NOSs (NOS1, NOS2 and NOS3). Typically, NOS1 and NOS3 produce NO at low or physiological levels. By contrast, NOS2 produces high levels of NO with a toxicity effect. Three isoforms of NOS were expressed increasingly in tumors and serve a dual role in tumor development as the effects of NO are strictly concentration-dependent (4). A study has demonstrated that low/intermediate concentrations of NO that slight higher than physiological dose was able to promote primary tumor growth and stimulate metastasis, whereas other studies revealed that metastasis was suppressed by higher levels of NO $(4,23)$. The present study demonstrated that NOS1 protein and mRNA were highly expressed in OVCAR3 cells. Griess assay revealed that the NO formed by NOSs was relatively low when compared with NOS1 with a concentration of $\sim 24 \mathrm{nM}$ in
OVCAR3 cell (5), therefore NOSs might serve a promoting role on a number of cellular functions, including cell proliferation, migration and invasion. However, the underlying molecular mechanism by which NOS1 promotes cell functions remains unclear and requires further study.

Chemotherapies are the most common treatments in advanced and recurrent ovarian cancer (24). The clinical response to cisplatin (DDP), one of first-line chemotherapeutic agents, is $\sim 80 \%$ in patients with ovarian cancer, but most patients with advanced disease will eventually relapse and succumb to the disease due to acquired drug resistance (25). A previous study demonstrated that combination therapies of DDP with other drugs were benefit to overcome drug-resistance and reduce toxicity (26). The present study identified that NOS1 expression contributed to DDP resistance in OVCAR3 cells, and inhibition of NOS1 by chemical inhibitor or NOS1 shRNA increased the sensitivity of cells to DDP-induced cell death, suggesting that therapeutic targeting of NOS1 in combination with conventional chemo-therapeutic agents may increase the efficacy of ovarian cancer therapy. In summary, the results of the present study suggested that NOS1 expression may be an indicator of response to chemotherapy. NOS1 may be an appropriate target for reducing chemoresistance to ovarian cancer therapy.

\section{Acknowledgements}

The authors would like to thank Dr Yaru Wang (Cancer Research Institute, Southern Medical University, Guangzhou, Guangdong, China) for the critical discussion during the experiments.

\section{Funding}

The present study was supported by the National Natural Science Foundation of China (grant no. 81472834), Guangzhou Science and Technology Research Project (grant no. 201400000001-1) and the Ministry of Education Key Laboratory Open Project (grant no. 2013jsz105).

\section{Availability of data and materials}

The datasets used and/or analyzed during the current study are available from the corresponding author on reasonable request.

\section{Authors' contributions}

ZWZ and XXL participated in the design of the study, performed all experiments and $\mathrm{ZWZ}$ wrote the manuscript. YLS, LQZ and ZZ assisted performing cells cultures, western blotting and invasion and migration assays. LLL, QBZ, MW and QLW helped with the statistical analysis. ZJL, BTH, YFW, YFP and KTY participcated in the design of the study. QZL conceived the study and assisted editing the manuscript. All authors read and approved the final manuscript.

\section{Ethics approval and consent to participate}

Not applicable. 


\section{Patient consent for publication}

Not applicable

\section{Competing interests}

The authors declare that they have no competing interests.

\section{References}

1. Rapozzi V, Della Pietra E and Bonavida B: Dual roles of nitric oxide in the regulation of tumor cell response and resistance to photodynamic therapy. Redox Biol 6: 311-317, 2015.

2. Fukumura D, Kashiwagi S and Jain RK: The role of nitric oxide in tumour progression. Nat Rev Cancer 6: 521-534, 2006.

3. Choudhari SK, Chaudhary M, Bagde S, Gadbail AR and Joshi V: Nitric oxide and cancer: A review. World J Surg Oncol 11: 118, 2013.

4. Ambs S and Glynn SA: Candidate pathways linking inducible nitric oxide synthase to a basal-like transcription pattern and tumor progression in human breast cancer. Cell Cycle 10: 619-624, 2011

5. Switzer CH, Glynn SA, Cheng RY, Ridnour LA, Green JE, Ambs S and Wink DA: S-nitrosylation of EGFR and Src activates an oncogenic signaling network in human basal-like breast cancer. Mol Cancer Res 10: 1203-1215, 2012.

6. Vannini F, Kashfi K and Nath N: The dual role of iNOS in cancer Redox Biol 6: 334-343, 2015

7. Wu X, Wang ZF, Xu Y, Ren R, Heng BL and Su ZX: Association between three eNOS polymorphisms and cancer risk: A meta-analysis. Asian Pac J Cancer Prev 15: 5317-5324, 2014.

8. Broholm H, Rubin I, Kruse A, Braendstrup O, Schmidt K, Skriver EB and Lauritzen M: Nitric oxide synthase expression and enzymatic activity in human brain tumors. Clin Neuropathol 22: 273-281, 2003

9. Ambs S, Bennett WP, Merriam WG, Ogunfusika MO, Oser SM, Khan MA, Jones RT and Harris CC: Vascular endothelial growth factor and nitric oxide synthase expression in human lung cancer and the relation to p53. Br J Cancer 78: 233-239, 1998.

10. Liu Y, Zhou R, Sulman EP, Scheurer ME, Boehling N, Armstrong GN, Tsavachidis S, Liang FW, Etzel CJ, Conrad CA, et al: Genetic modulation of neurocognitive function in glioma patients. Clin Cancer Res 21: 3340-3346, 2015.

11. Kotake M, Sato K, Mogi C, Tobo M, Aoki H, Ishizuka T, Sunaga N, Imai H, Kaira K, Hisada T, et al: Acidic $\mathrm{pH}$ increases cGMP accumulation through the OGR1/phospholipase $\mathrm{C} / \mathrm{Ca}(2+) /$ neuronal NOS pathway in N1E-115 neuronal cells. Cell Signal 26: 2326-2332, 2014.
12. Liu Q, Tomei S, Ascierto ML, De Giorgi V, Bedognetti D, Dai C, Uccellini L, Spivey T, Pos Z, Thomas J, et al: Melanoma NOS1 expression promotes dysfunctional IFN signaling. J Clin Invest 124: 2147-2159, 2014.

13. Hamaoka R, Yaginuma Y, Takahashi T, Fujii J, Koizumi M, Seo HG, Hatanaka Y, Hashizume K, Ii K, Miyagawa J, et al: Different expression patterns of nitric oxide synthase isozymes in various gynecological cancers. J Cancer Res Clin Oncol 125: 321-326, 1999.

14. Thomsen LL, Sargent JM, Williamson CJ and Elgie AW: Nitric oxide synthase activity in fresh cells from ovarian tumour tissue: Relationship of enzyme activity with clinical parameters of patients with ovarian cancer. Biochem Pharmacol 56: 1365-1370, 1998.

15. Caneba CA, Yang L, Baddour J, Curtis R, Win J, Hartig S, Marini J and Nagrath D: Nitric oxide is a positive regulator of the Warburg effect in ovarian cancer cells. Cell Death Dis 5: e1302, 2014.

16. Leung EL, Fraser M, Fiscus RR and Tsang BK: Cisplatin alters nitric oxide synthase levels in human ovarian cancer cells: Involvement in p53 regulation and cisplatin resistance. $\mathrm{Br} \mathrm{J}$ Cancer 98: 1803-1809, 2008.

17. Livak KJ and Schmittgen TD: Analysis of relative gene expression data using real-time quantitative PCR and the 2(-Delta Delta C(T)) method. Methods 25: 402-408, 2001.

18. Lawrie TA, Winter-Roach BA, Heus $\mathrm{P}$ and Kitchener HC Adjuvant (post-surgery) chemotherapy for early stage epithelial ovarian cancer. Cochrane Database Syst Rev 12: CD004706, 2015.

19. Cannistra SA: Cancer of the ovary. N Engl J Med 351: 2519-2529, 2004.

20. Park B, Park S, Kim TJ, Ma SH, Kim BG, Kim YM, Kim JW, Kang S, Kim J, Kim TJ, et al: Epidemiological characteristics of ovarian cancer in Korea. J Gynecol Oncol 21: 241-247, 2010.

21. Siegel R, Ward E, Brawley O and Jemal A: Cancer statistics, 2011: The impact of eliminating socioeconomic and racial disparities on premature cancer deaths. CA Cancer J Clin 61: 212-236, 2011.

22. Goff BA, Mandel L, Muntz HG and Melancon CH: Ovarian carcinoma diagnosis. Cancer 89: 2068-2075, 2000.

23. Vasudevan D and Thomas DD: Insights into the diverse effects of nitric oxide on tumor biology. Vitam Horm 96: 265-298, 2014.

24. Apps MG, Choi EH and Wheate NJ: The state-of-play and future of platinum drugs. Endocr Relat Cancer 22: R219-R233, 2015.

25. Dasari $S$ and Tchounwou PB: Cisplatin in cancer therapy: Molecular mechanisms of action. Eur J Pharmacol 740: 364-378, 2014.

26. Galluzzi L, Vitale I, Michels J, Brenner C, Szabadkai G, Harel-Bellan A, Castedo M and Kroemer G: Systems biology of cisplatin resistance: Past, present and future. Cell Death Dis 5: e1257, 2014. 